\title{
Editorial „Kommunikation und Koordination in Teams"
}

\section{Margarete Boos ${ }^{1} \mathbb{D} \cdot$ Michaela Kolbe}

Online publiziert: 20. Februar 2019

(c) Springer Fachmedien Wiesbaden GmbH, ein Teil von Springer Nature 2019

Wenn Teams eine gemeinsame Aufgabe erfolgreich lösen sollen, müssen sie ihre Informationen und Handlungen koordinieren. Dies gilt heute besonders auch für virtuelle Teamarbeit und stets für Aufgaben, deren Bewältigung nicht allein darin besteht, dass die Einzelleistungen der Teammitglieder - zum Beispiel ihre Ideen - additiv zu einem Gruppenergebnis zusammen gefügt werden. Schon bei solchen Ideenfindungsaufgaben treten neben Motivationsverlusten wie Trittbrettfahren Koordinationsverluste wie Produktionsblockierung auf (Diehl und Stroebe 1991; Stroebe und Diehl 1994). So erfordern schon einfache additive Aufgaben zumindest eine gemeinsame Orientierung am Ziel und eine zeitliche Synchronisation, also basale Koordination.

Folgt man der Definition von Koordination von Arrow et al. (2000) als aufgabenabhängiges Management der Interdependenzen individueller Ziele, Bedeutungszuschreibungen und Verhaltensweisen, wird klar, dass Koordinationsanforderungen sehr stark mit der Aufgabenstruktur variieren. Je komplexer die Gruppenaufgabe ist, desto mehr muss koordiniert werden (Boos et al. 2011, S. 14). So erfordert das Treffen einer Entscheidung in einem Team nicht nur die Abstimmung von Zielen und Aufgabenrepräsentationen unter den Teammitgliedern, sondern auch den Austausch und die Bewertung von Informationen und schließlich den Konsens über eine zu wählende Entscheidungsalternative.

Koordiniert wird in der Regel kommunikativ, es sei denn das Team verfügt „pre-process“ über Pläne, Routinen, Regeln oder Richtlinien, die diese Koordinationsfunktion erfüllen, ohne dass in der jeweils aktuellen Aufgabensituation ,in-process“ kommuniziert werden muss (Boos et al., 2011). So kann bei einer Routineoperation im Operationssaal lange Zeit implizit, also stillschweigend koordiniert werden, z. B. indem die instrumentierende Pflegekraft stets ohne dazu aufgefordert worden zu sein das richtige Instru-

Prof. Dr. Margarete Boos mboos@uni-goettingen.de

1 Institut für Psychologie, Universität Göttingen, Gosslerstr. 14, 37073 Göttingen, Deutschland ment für den/die Chirurg/-in anreicht. Sobald jedoch eine Komplikation oder ein unvorhergesehenes Ereignis eintritt, wird in der Regel explizit, d.h. ausdrücklich und in verbaler Kommunikation, koordiniert werden. So sollte beispielsweise im Team darüber gesprochen werden, worin die Komplikation besteht, was mögliche Ursachen sein können und welche Maßnahmen wann im Team ergriffen werden können, um zur Routine zurück kehren zu können.

Krenz, Burtscher und Kolbe beschreiben das Phänomen des ,voice behaviour“ in Teams. Es handelt sich dabei um ein Verhalten, in dem Verfahrensweisen, zum Beispiel bei einer Konstruktionsaufgabe, hinterfragt, korrigiert oder in dem auf Fehler hingewiesen wird. Dieses Verhalten erfordert Mut und Einsatz für die Qualität der Teamarbeit, vor allem, wenn es von Mitarbeitenden gezeigt wird, die einen niedrigeren Status im Team inne haben. Bisherige Studien $\mathrm{zu}$ voice behaviour (oder speaking up) fokussierten die Senderperspektive. Die Autor/-innen hier untersuchen, welche inhaltlichen und sprachlichen Mechanismen auf Senderseite Wirkung bei den Rezipient/-innen zeigen, gemessen an deren Bereitschaft, die Kritik umzusetzen.

Friedrichs und Ohly untersuchten die Frage, ob das Ausmaß, in dem Teammitglieder Aufgaben nur in Abhängigkeit von anderen Teammitgliedern bearbeiten können (Aufgabeninterdependenz) in studentischen Arbeitsteams den $\mathrm{Zu}$ sammenhang zwischen interpersonellen Prozessen und der Zufriedenheit des Teams beeinflusst. Als wichtige Teamprozesse werden dabei Konfliktmanagement, gegenseitige Motivation und die Regulation von Emotionen angesehen. Die Autor/-innen bestätigen, dass interpersonelle Prozesse und die Zufriedenheit der Teammitglieder positiv zusammen hängen, finden jedoch entgegen ihrer Vermutung nicht, dass höhere Aufgabeninterdependenz diesen Zusammenhang verstärkt. Dies legt die Frage nahe, inwiefern Aufgabeninterdependenzen geeignet sind, fehlende oder nicht gelingende interpersonelle Prozesse zu kompensieren und umgekehrt.

Müller und Antoni widmeten sich den Koordinationsanforderungen virtueller Teams. Sie diskutieren die Kompetenz des Teams im sinnvollen Umgang mit Medien und postulieren, dass virtuelle Teams ein gemeinsames Medi- 
enverständnis entwickeln müssen. Dieses gemeinsame Medienverständnis - eine Komponente des geteilten mentalen Modells - könne die potentiellen negativen Auswirkungen der Virtualität reduzieren.

Auch Handke und Kauffeld beschäftigten sich mit den Koordinationsherausforderungen virtueller Teams. Am Beispiel eines Softwareentwicklungsteams demonstrieren sie im Detail, vor welchen Schwierigkeiten virtuelle Teams stehen. Abschließend geben sie konkrete Handlungsempfehlungen, die für die erfolgreiche Zusammenarbeit in virtuellen Teams zentral sind.

Ufer berichtet über das Praxisbeispiel der Beratung einer Nationalmannschaft im Sport, welche sich über drei Monate intensiver Arbeit mit Mannschaft und Trainern erstreckte. Ansätze und Methoden der Beratung werden offen gelegt. Der Beratungsauftrag durch den Bundestrainer lautete, die Motivation und die Leistungsfähigkeit des Teams zu steigern, um die Qualifikation zur Europameisterschaft zu erreichen. Es wird in diesem Beispiel gezeigt, wie sich im Laufe der Beratung die Perspektive auf die Kommunikation und Koordination zwischen Mannschaft und Betreuer sowie das Thema Führung erweiterte.

Rack, Zahn und Bleisch stellen den Ansatz der ,visual analytics“ dar, mit dem multidimensionale Daten mit hierarchisch-sequentieller Struktur, wie sie häufig im Rahmen empirischer Studien zu Koordinations- und Kommunikationsprozessen anfallen, visuell dargestellt werden können. Anhand verschiedener Forschungsbeispiele wird gezeigt, wie visuelle Metaphern und Darstellungsformen mit zugrundeliegenden Algorithmen zur Datenanalyse verknüpft und sowohl explorativ als auch konfirmatorisch verwendet werden können.

Die Beiträge des offenen Teils thematisieren ebenfalls Gruppenprozesse in einem weiteren Sinne. Schuster und Lobnig zeigen, wie sich im Rahmen von Marketingaktivitäten die so unterschiedlichen Gewohnheiten und Ansätze von Vertrieb und Technik ergänzen können. Klasmeier, Pachocki und Rowold beschäftigen sich aus psychohistorischer Perspektive mit dem Zusammenhang zwischen Führungsverhalten und Emotionen. Göritz und Rennung beschreiben ein längsschnittliches Feldexperiment, in dem sie die Auswirkungen einer Intervention, die aus synchroner Bewegung im Team bestand, auf die Teamkohäsion, das Stressempfinden der Teammitglieder und krankheitsbedingte Abwesenheit untersuchten.

Mit dem Heft versuchen wir, wissenschaftliche und praktische Einblicke in Prozesse der Koordination und Kommunikation in Teams zu gewinnen, die die Verschiedenartigkeit von Teams erfassen und gleichzeitig Muster und RegelmäBigkeiten identifizieren helfen. In den einzelnen Beiträgen werden auch unterschiedliche methodische Herangehensweisen demonstriert, wie Koordinations- und Kommunikationsprozesse im Team messbar und visualisierbar gemacht werden können.

\section{Literatur}

Arrow, H., McGrath, J.E., \& Berdahl, J.L. (2000). Small groups as complex systems: Formation, coordination, development, and adaption. Thousand Oaks: SAGE.

Boos, M., Kolbe, M., \& Strack, M. (2011). An inclusive model of group coordination. In M. Boos, M. Kolbe, P. M. Kappeler \& T. Ellwart (Hrsg.), Coordination in human and primate groups (S. 11-35). Berlin: Springer.

Diehl, M., \& Stroebe, W. (1991). Productivity loss in idea-generating groups: Tracking down the blocking effect. Journal of Personality and Social Psychology, 61, 392-403.

Stroebe, W., \& Diehl, M. (1994). Why groups are less effective than their members: On productivity losses in idea-generating groups. European Review of Social Psychology, 2, 271-303.

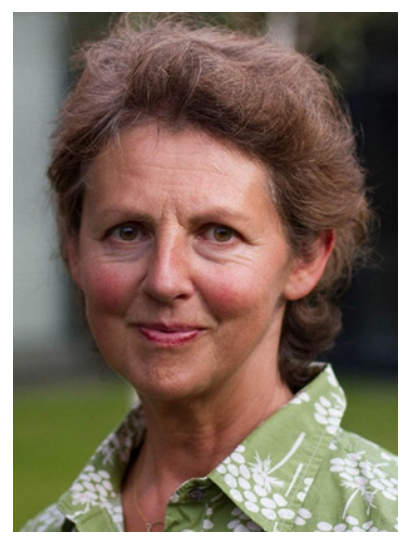

Prof. Dr. Margarete Boos hat einen Lehrstuhl für Wirtschafts- und Sozialpsychologie an der Universität Göttingen inne. Sie beschäftigt sich in Forschung und Lehre mit folgenden Schwerpunkten: Führung verteilter Teams, Koordination und Führung in medizinischen Crews, computervermittelte Kommunikation und Methoden der Interaktionsund Kommunikationsanalyse. Sie setzt sozial- und kommunikationspsychologische Forschungsergebnisse in Methoden zur Teamdiagnostik und zum Teamtraining um und hat gemeinsam mit Kolleg/innen aus den Wirtschaftswissenschaften 2010 das Unternehmen Malamut Team Catalyst GmbH (http://www.malamutteamcatalyst.de/) gegründet.

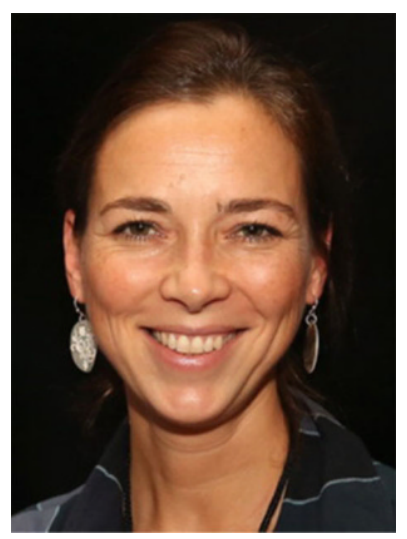

PD Dr. Michaela Kolbe beschäftigt sich seit über 10 Jahren mit Teamprozessen in der Medizin und hat dazu zahlreiche Forschungsprojekte durchgeführt. Sie interessiert sich besonders dafür, wie Mitarbeitende im Krankenhaus trotz Hierarchie und über Disziplingrenzen hinaus sicherheitsrelevante Bedenken offen ansprechen können. Michaela Kolbe ist Psychologin und leitet das Simulationszentrum des Universitätsspitals Zürich. 